\title{
On the Ginzburg-Landau energy with weight
}

\author{
by
}

\author{
Cătălin LEFTER ${ }^{1,2}$ and Vicenţiu D. RĂDULESCU ${ }^{1,3}$ \\ ${ }^{1}$ Laboratoire d'Analyse Numérique, Tour 55-65, \\ Université Pierre et Marie Curie, \\ 4 , place Jussieu, \\ 75252 Paris, France. \\ ${ }^{2}$ Department of Mathematics, \\ University of Iaşi, \\ 6600 Iaşi, Romania. \\ ${ }^{3}$ Department of Mathematics, \\ University of Craiova, \\ 1100 Craiova, Romania.
}

\begin{abstract}
This paper gives a solution to an open problem raised by Bethuel, Brezis and Hélein. We study the Ginzburg-Landau energy with weight. We find the expression of the renormalized energy and we show that the finite configuration of singularities of the limit is a minimum point of this functional. We find a vanishing gradient type property and then we obtain the renormalized energy by Bethuel, Brezis and Hélein's shrinking holes method.
\end{abstract}

Key words: Ginzburg-Landau energy with weight, renormalized energy.

RÉsumÉ. - Ce travail donne la solution d'un problème ouvert de Bethuel, Brezis et Hélein. On étudie l'énergie de Ginzburg-Landau avec poids. Nous trouvons l'expression de l'énergie renormalisée et on prouve que la configuration finie des singularités de la limite est un point de minimum pour cette fonctionnelle. Nous montrons une propriété du type «vanishing gradient » et on obtient ensuite l'énergie renormalisée avec la méthode « shrinking holes» de Bethuel, Brezis et Hélein.

Classification A.M.S. : 35 J 60, 35 Q 99. 


\section{INTRODUCTION}

In a recent book [BBH4], F. Bethuel, H. Brezis and F. Hélein studied the vortices related to the Ginzburg-Landau functional. Similar functionals appear in the study of problems occuring in superconductivity or the theory of superfluids.

In [BBH4], F. Bethuel, H. Brezis and F. Hélein have studied the behavior as $\varepsilon \rightarrow 0$ of minimizers $u_{\varepsilon}$ of the Ginzburg-Landau energy

$$
E_{\varepsilon}(u)=\frac{1}{2} \int_{G}|\nabla u|^{2}+\frac{1}{4 \varepsilon^{2}} \int_{G}\left(1-|u|^{2}\right)^{2}
$$

in the class of functions

$$
H_{g}^{1}(G)=\left\{u \in H^{1}\left(G ; \mathbb{R}^{2}\right) ; u=g \text { on } \partial G\right\},
$$

where:

a) $\varepsilon>0$ is a (small) parameter.

b) $G$ is a smooth, simply connected, starshaped domain in $\mathbb{R}^{2}$.

c) $g: \partial G \rightarrow S^{1}$ is a smooth data with a topological degree $d>0$.

They obtained the convergence of $\left(u_{\varepsilon_{n}}\right)$ in certain topologies to $u_{\star}$. The function $u_{\star}$ is a harmonic map from $G \backslash\left\{a_{1}, \ldots, a_{d}\right\}$ to $S^{1}$, and is canonical, in the sense that

$$
\frac{\partial}{\partial x_{1}}\left(u_{\star} \wedge \frac{\partial u_{\star}}{\partial x_{1}}\right)+\frac{\partial}{\partial x_{2}}\left(u_{\star} \wedge \frac{\partial u_{\star}}{\partial x_{2}}\right)=0 \text { in } \mathcal{D}^{\prime}(G) .
$$

Recall (see [BBH4]) that a canonical harmonic map $u_{\star}$ with values in $S^{1}$ and singularities $b_{1}, \ldots, b_{k}$ of degrees $d_{1}, \ldots, d_{k}$ may be expressed as

$$
u_{\star}(x)=\left(\frac{x-b_{1}}{\left|x-b_{1}\right|}\right)^{d_{1}} \cdots\left(\frac{x-b_{k}}{\left|x-b_{k}\right|}\right)^{d_{k}} e^{i \varphi_{0}(x)}
$$

with

$$
\Delta \varphi_{0}=0 \quad \text { in } G .
$$

They also defined the notion of renormalized energy $W(b, \bar{d}, g)$ associated to a given configuration $b=\left(b_{1}, \ldots, b_{k}\right)$ of distinct points with associated degrees $\bar{d}=\left(d_{1}, \ldots, d_{k}\right)$. For simplicity we set $W(b)=W(b, \bar{d}, g)$ when $k=d$ and all the degrees equal +1 . The expression of the renormalized energy $W$ is given by

$$
W(b, \bar{d}, g)=-\pi \sum_{i \neq j} d_{i} d_{j} \log \left|b_{i}-b_{j}\right|+\frac{1}{2} \int_{\partial G} \Phi_{0}\left(g \wedge g_{\tau}\right)-\pi \sum_{j=1}^{k} d_{j} R_{0}\left(b_{j}\right),
$$


where $\Phi_{0}$ is the unique solution of

$$
\left\{\begin{array}{l}
\Delta \Phi_{0}=2 \pi \sum_{j=1}^{k} d_{j} \delta_{b_{j}}, \quad \text { in } G \\
\frac{\partial \Phi_{0}}{\partial \nu}=g \wedge g_{\tau}, \quad \text { on } \partial G \\
\int_{\partial G} \Phi_{0}=0
\end{array}\right.
$$

and

$$
R_{0}(x)=\Phi_{0}(x)-\sum_{j=1}^{k} d_{j} \log \left|x-b_{j}\right| .
$$

The functional $W$ is also related to the asymptotic behavior of minimizers $u_{\varepsilon}$ as follows:

$$
\lim _{\varepsilon \rightarrow 0}\left\{E_{\varepsilon}\left(u_{\varepsilon}\right)-\pi d|\log \varepsilon|\right\}=\min _{b \in G^{d}} W(b)+d \gamma,
$$

where $\gamma$ is an universal constant, $k=d, d_{i}=+1$ for all $i$ and the configuration $a=\left(a_{1}, \cdots, a_{d}\right)$ achieves the minimum of $W$.

We study in this paper a similar problem, related to the Ginzburg-Landau energy with the weight $w$, that is

$$
E_{\varepsilon}^{w}(u)=\frac{1}{2} \int_{G}|\nabla u|^{2}+\frac{1}{4 \varepsilon^{2}} \int_{G}\left(1-|u|^{2}\right)^{2} w
$$

with $w \in C^{1}(\bar{G}), w>0$ in $\bar{G}$. Throughout, $u_{\varepsilon}$ will denote a minimizer of $E_{\varepsilon}^{w}$. We mention that $u_{\varepsilon}$ verifies the Ginzburg-Landau equation with weight

$$
\left\{\begin{array}{l}
-\Delta u_{\varepsilon}=\frac{1}{\varepsilon^{2}} u_{\varepsilon}\left(1-\left|u_{\varepsilon}\right|^{2}\right) w \text { in } G \\
u_{\varepsilon}=g \text { on } \partial G .
\end{array}\right.
$$

Our work is motivated by the Open Problem 2, p. 137 in [BBH4]. We are concerned in this paper with the study of the convergence of minimizers, as well as with the corresponding expression of the renormalized energy. We prove that the behavior of minimizers is of the same type as in the case $w \equiv 1$, the change appearing in the expression of the renormalized energy and, consequently, in the location of singularities of the limit $u_{\star}$ of $u_{\varepsilon_{n}}$. In our proof we borrow some of the ideas from Chapter VIII in [BBH4], without relying on the vanishing gradient property that is used there. We then prove a corresponding vanishing gradient property for the configuration of singularities obtained at the limit. In the last section we obtain the new renormalized energy by a variant of the "shrinking holes" method which was developed in [BBH4], Chapter I. 


\section{THE RENORMALIZED ENERGY}

THEOREM 1. - There is a sequence $\varepsilon_{n} \rightarrow 0$ and exactly $d$ points $a_{1}, \ldots, a_{d}$ in $G$ such that

$$
u_{\epsilon_{n}} \rightarrow u_{\star} \text { in } H_{\mathrm{loc}}^{1}\left(\bar{G} \backslash\left\{a_{1}, \ldots, a_{d}\right\} ; \mathbb{R}^{2}\right),
$$

where $u_{\star}$ is the canonical harmonic map associated to the singularities $a_{1}, \ldots, a_{d}$ of degrees +1 and to the boundary data $g$.

Moreover, $a=\left(a_{1}, \cdots, a_{d}\right)$ minimizes the functional

$$
\widetilde{W}(b)=W(b)+\frac{\pi}{2} \sum_{j=1}^{d} \log w\left(b_{j}\right)
$$

among all configurations $b=\left(b_{1}, \ldots, b_{d}\right)$ of d distinct points in $G$.

In addition, the following holds:

(5) $\lim _{n \rightarrow \infty}\left\{E_{\varepsilon_{n}}^{w}\left(u_{\varepsilon_{n}}\right)-\pi d\left|\log \varepsilon_{n}\right|\right\}=W(a)+\frac{\pi}{2} \sum_{j=1}^{d} \log w\left(a_{j}\right)+d \gamma$,

where $\gamma$ is some universal constant, the same as in (2).

Remark. - The functional $\widetilde{W}$ may be regarded as the renormalized energy corresponding to the energy $E_{\varepsilon}^{w}$.

Before giving the proof, we shall make some useful notations: given the constants $c, \varepsilon, \eta>0$, set

$$
I^{c}(\varepsilon, \eta)=\min \left\{E_{\varepsilon}^{c}(u) ; u \in H^{1}\left(B_{\eta} ; \mathbb{R}^{2}\right) \text { and } u(x)=\frac{x}{\eta} \text { on } \partial B_{\eta}\right\} .
$$

Here $B_{\eta}=B(0, \eta) \subset \mathbb{R}^{2}$.

For $x \in G$, denote

$$
M_{\eta}(x)=\sup _{B(x, \eta) \cap \bar{G}} w \quad \text { and } \quad m_{\eta}(x)=\inf _{B(x, \eta) \cap \bar{G}} w .
$$

Note that

$$
I^{c}(\varepsilon, \eta)=I^{c}\left(\frac{\varepsilon}{\eta}, 1\right)=I^{1}\left(\frac{\varepsilon}{\eta \sqrt{c}}, 1\right)
$$

and

$$
I^{c_{1}}(\varepsilon, \eta) \leq I^{c_{2}}(\varepsilon, \eta)
$$

provided $c_{1} \leq c_{2}$. 
We shall drop the superscript $c$ if it equals 1 .

Proof of Theorem 1. - The first part of the conclusion may be obtained by adapting the techniques developed in [BBH1], [BBH2], [BBH3], [BBH4] (see also $[\mathrm{S}]$ ). We shall point out only the main steps that are necessary to prove the convergence:

a) Using the techniques from [S] we find a sequence $\varepsilon_{n} \rightarrow 0$ such that, for each $n$,

$$
\frac{1}{\varepsilon_{n}^{2}} \int_{G}\left(1-\left|u_{\varepsilon_{n}}\right|^{2}\right)^{2} w \leq C
$$

b) Using the methods developed in [BBH4], Chapters 3-5, we determine the "bad" disks, as well as the fact that their number is uniformly bounded. These techniques allow us to prove the convergence of $\left(u_{\varepsilon_{n}}\right)$ weakly in $H_{\text {loc }}^{1}\left(G \backslash\left\{a_{1}, \ldots, a_{k}\right\} ; \mathbb{R}^{2}\right)$ to $u_{\star}$, which is the canonical harmonic map associated to $a_{1}, \ldots, a_{k}$ with some degrees $d_{1}, \ldots, d_{k}$ and to the given boundary data.

c) The strong convergence of $\left(u_{\varepsilon_{n}}\right)$ in $H_{\text {loc }}^{1}\left(\bar{G} \backslash\left\{a_{1}, \ldots, a_{k}\right\} ; \mathbb{R}^{2}\right)$ follows as in [BBH4], Theorem VI.1 with the techniques from [BBH3], Theorem 2, Step 1. Now the local convergence of $\left(u_{\varepsilon_{n}}\right)$ in $G \backslash\left\{a_{1}, \cdots, a_{k}\right\}$ in stronger topologies, say $C^{2}$, may be easily obtained by a bootstrap argument in (3). This implies that

$$
\frac{1-\left|u_{\varepsilon_{n}}\right|^{2}}{\varepsilon_{n}^{2}} w \rightarrow\left|\nabla u_{\star}\right|^{2}
$$

uniformly on every compact subset of $G \backslash\left\{a_{1}, \ldots, a_{k}\right\}$.

d) For each $1 \leq j \leq k, \operatorname{deg}\left(u_{\star}, a_{j}\right) \neq 0$. Indeed, if not, then as in Step 1 of Theorem 2 [BBH3], the $H^{1}$-convergence is extended up to $a_{j}$, which becomes a "removable singularity".

e) The fact that all degrees equal +1 may be deduced as in Theorem VI.2, [BBH4].

f) The points $a_{1}, \ldots, a_{d}$ lie in $G$. The proof of this fact is similar to the corresponding result in [BBH4].

The proof of the second part of the theorem is divided into 3 steps:

Step 1. - An upper bound for $E_{\varepsilon}^{w}\left(u_{\varepsilon}\right)$.

We shall prove that if $b=\left(b_{j}\right)$ is an arbitrary configuration of $d$ distinct points in $G$, then there exists $\eta_{0}>0$ such that, for each $\eta<\eta_{0}$,

$$
\begin{aligned}
E_{\varepsilon}^{w}\left(u_{\varepsilon}\right) \leq & \sum_{j=1}^{d} I\left(\frac{\varepsilon}{\eta \sqrt{M_{\eta}\left(b_{j}\right)}}, 1\right)+W(b) \\
& +\pi d \log \frac{1}{\eta}+O(\eta) \quad \text { as } \eta \rightarrow 0
\end{aligned}
$$


for $\varepsilon>0$ small enough. Here $O(\eta)$ is a quantity which is bounded by $C \eta$, with $C$ independent of $\eta>0$ small enough.

The idea is to construct a suitable comparison function $v_{\varepsilon}$. Let $\eta<\eta_{0}$, where

$$
\eta_{0}=\min _{j, k}\left\{\operatorname{dist}\left(b_{j}, \partial G\right),\left|b_{j}-b_{k}\right|\right\} .
$$

Applying Theorem I.9 in [BBH4] to the configuration $b$, we find $\widetilde{u}: G_{\eta}:=G \backslash \bigcup_{j=1}^{d} \overline{B\left(b_{j}, \eta\right)} \rightarrow S^{1}$ with $\widetilde{u}=g$ on $\partial G$ and $\alpha_{j} \in \mathbb{C}$, $\left|\alpha_{j}\right|=1$ such that

$$
\widetilde{u}=\alpha_{j} \frac{z-b_{j}}{\left|z-b_{j}\right|} \quad \text { on } \partial B\left(b_{j}, \eta\right)
$$

and

$$
\frac{1}{2} \int_{G_{\eta}}|\nabla \widetilde{u}|^{2}=\pi d \log \frac{1}{\eta}+W(b)+O(\eta), \quad \text { as } \eta \rightarrow 0
$$

We define $v_{\varepsilon}$ as follows: let $v_{\varepsilon}=\widetilde{u}$ on $G_{\eta}$ and, in $B\left(b_{j}, \eta\right)$, let $v_{\varepsilon}$ be a minimizer of $E_{\varepsilon}^{w}$ on $H_{h}^{1}\left(B\left(b_{j}, \eta\right) ; \mathbb{R}^{2}\right)$, where $h=\left.\widetilde{u}\right|_{\partial B\left(b_{j}, \eta\right)}$. We have the following estimate

$$
E_{\varepsilon}^{w}\left(\left.v_{\varepsilon}\right|_{B\left(b_{j}, \eta\right)}\right) \leq I^{M_{\eta}\left(b_{j}\right)}(\varepsilon, \eta)=I\left(\frac{\varepsilon}{\eta \sqrt{M_{\eta}\left(b_{j}\right)}}, 1\right) .
$$

The desired conclusion follows from (9), (10) and $E_{\varepsilon}^{w}\left(u_{\varepsilon}\right) \leq E_{\varepsilon}^{w}\left(v_{\varepsilon}\right)$.

Step 2. - A lower bound for $E_{\varepsilon_{n}}^{w}\left(u_{\varepsilon_{n}}\right)$.

We shall prove that, if $a_{1}, \ldots, a_{d}$ are the singularities of $u_{\star}$, then given any $\eta>0$, there is $N_{0}=N_{0}(\eta) \in \mathbb{N}$ such that, for each $n \geq N_{0}$,

$$
E_{\varepsilon_{n}}^{w}\left(u_{\varepsilon_{n}}\right) \geq \sum_{j=1}^{d} I\left(\frac{\varepsilon_{n}}{\alpha \eta \sqrt{m_{\alpha \eta}\left(a_{j}\right)}}, 1\right)+\pi d \log \frac{1}{\eta}+W(a)+O(\eta)
$$

Here $\alpha=1+\eta$ and $O(\eta)$ is a quantity with the same behavior as in (8).

Indeed, for a fixed $a_{j}$, supposed to be $0, u_{\star}$ may be written

$$
u_{\star}=e^{i(\psi+\theta)},
$$

where $\psi$ is a smooth harmonic function in a neighbourhood of 0 . We may assume, without loss of generality, that $\psi(0)=0$. 
In the annulus $A_{\eta, \alpha \eta}=\left\{x \in \mathbb{R}^{2} ; \eta \leq|x| \leq \alpha \eta\right\}$ the function $u_{\varepsilon_{n}}$ may be written, for $n$ large enough, as

$$
u_{\varepsilon_{n}}=\rho_{n} e^{i\left(\psi_{n}+\theta\right)}
$$

where $\psi_{n}$ is a smooth function and $0<\rho_{n} \leq 1$. Define, for $\eta \leq r \leq \alpha \eta$, the interpolation function

$$
v_{n}(r, \theta)=\frac{r-\eta+\rho_{n}(\eta, \theta)(\alpha \eta-r)}{\eta(\alpha-1)} \cdot e^{i\left[\frac{\alpha \eta-r}{\eta(\alpha-1)} \psi_{n}(\eta, \theta)+\theta\right]}
$$

We have

$$
\begin{gathered}
\frac{1}{\varepsilon_{n}^{2}} \int_{A_{\eta, \alpha \eta}}\left(1-\left|v_{n}\right|^{2}\right)^{2} w \leq \frac{\|w\|_{L^{\infty}}}{\varepsilon_{n}^{2}} \cdot \int_{\eta}^{\alpha \eta} \frac{r}{\eta}\left(\int_{\partial B_{\eta}}\left(1-\left|u_{n}\right|^{2}\right)^{2} d \sigma\right) d r \\
=\|w\|_{L^{\infty}} \cdot \frac{\alpha+1}{2} \eta^{2} \int_{\partial B_{\eta}} \frac{\left(1-\left|u_{n}\right|^{2}\right)^{2}}{\varepsilon_{n}^{2}} d \sigma \rightarrow 0, \quad \text { as } n \rightarrow \infty
\end{gathered}
$$

This convergence is motivated by (7). We also observe that the convergence of $\left(u_{\varepsilon_{n}}\right)$ in $H_{\text {loc }}^{1}\left(G \backslash\left\{a_{1}, \ldots, a_{d}\right\} ; \mathbb{R}^{2}\right)$ implies

$$
\int_{A_{\eta, \alpha \eta}}\left|\nabla v_{n}\right|^{2} \rightarrow \int_{A_{\eta, \alpha \eta}}|\nabla v|^{2}, \quad \text { as } \eta \rightarrow 0
$$

where

$$
v(\eta, \theta)=e^{i\left[\frac{\alpha \eta-r}{\eta(\alpha-1)} \psi(\eta, \theta)+\theta\right]} .
$$

Thus, we may write, for $n \geq N_{1}$,

$$
E_{\varepsilon_{n}}^{w}\left(v_{n} \mid A_{\eta, \alpha \eta}\right)=\frac{1}{2} \int_{A_{\eta, \alpha \eta}}|\nabla v|^{2}+o(1) .
$$

We prove in what follows that

$$
\int_{A_{\eta, \alpha \eta}}|\nabla v|^{2}=O(\eta)
$$

Indeed, since

$$
|\nabla v|^{2}=\frac{\psi^{2}(\eta, \theta)}{\eta^{2}(\alpha-1)^{2}}+\frac{1}{r^{2}}\left[\frac{\alpha \eta-r}{\eta(\alpha-1)} \psi_{\theta}(\eta, \theta)+1\right]^{2}
$$

and

$$
\psi(r, \theta) \leq C r, \quad\left|\psi_{r}(r, \theta)\right| \leq C, \quad\left|\psi_{\theta}(r, \theta)\right| \leq C r,
$$

the desired conclusion follows by a straightforward calculation. 
We obtain

$$
E_{\varepsilon_{n}}^{w}\left(v_{\left.\varepsilon_{n}\right|_{B}\left(a_{j}, \eta\right)}\right) \geq I^{m_{\alpha \eta}\left(a_{j}\right)}\left(\varepsilon_{n}, \alpha \eta\right)+O(\eta)
$$

On the other hand, by the convergence of $\left(u_{\varepsilon_{n}}\right)$ in $H_{\mathrm{loc}}^{1}(G)$ $\left.\left\{a_{1}, \ldots, a_{d}\right\} ; \mathbb{R}^{2}\right)$ it follows that

$$
E_{\varepsilon_{\pi}}^{w}\left(\left.u_{\varepsilon_{n}}\right|_{G_{\eta}}\right)=\int_{G_{\eta}}\left|\nabla u_{\star}\right|^{2}+O(\eta)
$$

for $\varepsilon_{n}$ sufficiently small.

Taking into account (12)-(15) we obtain the desired result.

Step 3. - The final conclusion.

It follows from [BBH4], Chapter IX that

$$
I(\varepsilon, \eta)=\pi\left|\log \frac{\varepsilon}{\eta}\right|+\gamma+o(1) \quad \text { as } \quad \frac{\varepsilon}{\eta} \rightarrow 0
$$

where the constant $\gamma$ represents the minimum of the renormalized energy corresponding to the boundary data $x$ in $B_{1}$.

From (8) and (11) we obtain

$$
W(b)+\frac{\pi}{2} \sum_{j=1}^{d} \log \underline{M}_{\eta}\left(b_{j}\right)-\pi d \log \varepsilon_{n}+d \gamma+o(1)
$$

$\geq W(a)+\frac{\pi}{2} \sum_{i=1}^{d} \log m_{\eta}\left(a_{i}\right)-\pi d \log \varepsilon_{n}+\pi d \log \frac{1}{\eta}-\pi d \log \frac{1}{\eta}+d \gamma+o(1)$,

where $o(1)$ stands for a quantity which goes to 0 as $\varepsilon_{n} \rightarrow 0$ for fixed $\eta$. Adding $\pi d \log \varepsilon_{n}$ and passing to the limit firstly as $n \rightarrow \infty$ and then as $\eta \rightarrow 0$, we obtain that $a=\left(a_{1}, \ldots, a_{d}\right)$ is a global minimum point of $\widetilde{W}$. We also deduce that

$$
\lim _{n \rightarrow \infty}\left\{E_{\varepsilon_{n}}^{w}\left(u_{\varepsilon_{n}}\right)-\pi d\left|\log \varepsilon_{n}\right|\right\}=W(a)+\frac{\pi}{2} \sum_{j=1}^{d} \log w\left(a_{j}\right)+d \gamma
$$

We now generalize another result from [BBH4] concerning the behavior of $u_{\varepsilon}$. 
THEOREM 2. - Set

$$
W_{n}=\frac{1}{4 \varepsilon_{n}^{2}}\left(1-\left|u_{\varepsilon_{n}}\right|^{2}\right)^{2} w .
$$

Then $\left(W_{n}\right)$ converges in the weak $\star$ topology of $C(\bar{G})$ to

$$
W_{\star}=\frac{\pi}{2} \sum_{j=1}^{d} \delta_{a_{j}} .
$$

Proof. - The boundedness of $\left(W_{n}\right)$ in $L^{1}(G)$ follows directly from (6). Hence (up to a subsequence), $W_{n}$ converges in the sense of measures of $\bar{G}$ to some $W_{\star}$. With the same techniques as those developed in [BBH3] (Theorem 2) or [BBH4] (Theorem X.3) we can obtain that, for any compact subset $K$ of $\bar{G} \backslash \bigcup_{j=1}^{d}\left\{a_{j}\right\}$,

Hence

$$
\frac{1}{\varepsilon_{n}^{2}}\left\|1-\left|u_{\varepsilon_{n}}\right|^{2}\right\|_{L^{\infty}(K)} \leq C_{K} .
$$

Therefore

$$
\operatorname{supp} W_{\star} \subset \bigcup_{j=1}^{d}\left\{a_{j}\right\} \text {. }
$$

$$
W_{\star}=\sum_{j=1}^{d} m_{j} \delta_{a_{j}} \quad \text { with } m_{j} \in \mathbb{R} .
$$

We now determine $m_{j}$ using the same methods as in [BBH4]. Fix one of the points $a_{j}$ (supposed to be 0 ) and consider $B_{R}=B(0, R)$ for $R$ small enough so that $B_{R}$ contains no other point $a_{i}(i \neq j)$. As in the proof of the Pohozaev identity, multiplying the Ginzburg-Landau equation (3) by $x \cdot \nabla u_{\varepsilon}$ and integrating on $B_{R}$ we obtain

$$
\begin{aligned}
\frac{R}{2} \int_{\partial B_{R}}\left|\frac{\partial u_{\varepsilon}}{\partial \nu}\right|^{2} & +\frac{1}{2 \varepsilon^{2}} \int_{B_{R}}\left(1-\left|u_{\varepsilon}\right|^{2}\right)^{2} w+\frac{1}{4 \varepsilon^{2}} \int_{B_{R}}\left(1-\left|u_{\varepsilon}\right|^{2}\right)^{2}(\nabla w \cdot x) \\
& =\frac{R}{2} \int_{\partial B_{R}}\left|\frac{\partial u_{\varepsilon}}{\partial \tau}\right|^{2}+\frac{R}{4 \varepsilon^{2}} \int_{\partial B_{R}}\left(1-\left|u_{\varepsilon}\right|^{2}\right)^{2} w
\end{aligned}
$$

Passing to the limit in (18) as $\varepsilon \rightarrow 0$ and using the convergence of $W_{n}$ we find

$$
\frac{R}{2} \int_{\partial B_{R}}\left|\frac{\partial u_{\star}}{\partial \nu}\right|^{2}+2 m_{j}=\frac{R}{2} \int_{\partial B_{R}}\left|\frac{\partial u_{\star}}{\partial \tau}\right|^{2} .
$$


Using now the expression of $u_{\star}$ around a singularity we deduce that, on $\partial B_{R}$,

$$
\begin{gathered}
\left|\frac{\partial u_{\star}}{\partial \nu}\right|^{2}=\left|\frac{\partial \theta}{\partial \nu}+\frac{\partial \psi}{\partial \nu}\right|^{2}=\left|\frac{\partial \psi}{\partial \nu}\right|^{2} \\
\left|\frac{\partial u_{\star}}{\partial \tau}\right|^{2}=\left|\frac{\partial \theta}{\partial \tau}+\frac{\partial \psi}{\partial \tau}\right|^{2}=\frac{1}{R^{2}}+\frac{2}{R} \frac{\partial \psi}{\partial \tau}+\left|\frac{\partial \psi}{\partial \tau}\right|^{2} .
\end{gathered}
$$

Inserting (20) and (21) into (19) we obtain

$$
\frac{R}{2} \int_{\partial B_{R}}\left|\frac{\partial \psi}{\partial \nu}\right|^{2}+2 m_{j}=\pi+\frac{R}{2} \int_{\partial B_{R}}\left|\frac{\partial \psi}{\partial \tau}\right|^{2} .
$$

On the other hand, multiplying $\Delta \psi=0$ by $x \cdot \nabla \psi$ and integrating on $B_{R}$ we find

$$
\frac{R}{2} \int_{\partial B_{R}}\left|\frac{\partial \psi}{\partial \nu}\right|^{2}=\frac{R}{2} \int_{\partial B_{R}}\left|\frac{\partial \psi}{\partial \tau}\right|^{2} .
$$

Thus, from (17) and (18) we obtain

$$
m_{j}=\frac{\pi}{2}
$$

\section{THE VANISHING GRADIENT PROPERTY OF THE RENORMALIZED ENERGY WITH WEIGHT}

The expression of the renormalized energy $\widetilde{W}$ allows us, by using the results obtained in [BBH4], to give an expression of the vanishing gradient property in the case of a weight.

From (4) it follows that

$$
D \widetilde{W}\left(b_{1}, \ldots, b_{d}\right)=D W\left(b_{1}, \ldots, b_{d}\right)+\frac{\pi}{2}\left(\frac{\nabla w\left(b_{1}\right)}{w\left(b_{1}\right)}, \ldots, \frac{\nabla w\left(b_{d}\right)}{w\left(b_{d}\right)}\right),
$$

for each configuration $b=\left(b_{1}, \ldots, b_{d}\right) \in G^{d}$.

Recall now Theorem VIII.3 in [BBH4], which gives the expression of the differential of $W$ in an arbitrary configuration of distinct points 
$b=\left(b_{1}, \ldots, b_{d}\right) \in G^{d}$

$$
\begin{aligned}
& D W(b)=-2 \pi\left[\left(\frac{\partial S_{1}}{\partial x_{1}}\left(b_{1}\right), \frac{\partial S_{1}}{\partial x_{2}}\left(b_{1}\right)\right), \ldots,\left(\frac{\partial S_{d}}{\partial x_{1}}\left(b_{d}\right), \frac{\partial S_{d}}{\partial x_{2}}\left(b_{d}\right)\right)\right] \\
& =2 \pi\left[\left(-\frac{\partial H_{1}}{\partial x_{2}}\left(b_{1}\right), \frac{\partial H_{1}}{\partial x_{1}}\left(b_{1}\right)\right), \ldots,\left(-\frac{\partial H_{d}}{\partial x_{2}}\left(b_{d}\right), \frac{\partial H_{d}}{\partial x_{1}}\left(b_{d}\right)\right)\right] .
\end{aligned}
$$

Here $S_{j}(x)=\Phi_{0}(x)-\log \left|x-b_{j}\right|$ in $G$ and $\Phi_{0}$ the unique solution of

$$
\left\{\begin{array}{l}
\Delta \Phi_{0}=2 \pi \sum_{j=1}^{d} \delta_{b_{j}}, \quad \text { in } G \\
\frac{\partial \Phi_{0}}{\partial \nu}=g \wedge g_{\tau}, \quad \text { on } \partial G \\
\int_{\partial G} \Phi_{0}=0 .
\end{array}\right.
$$

The function $H_{j}$ is harmonic around $b_{j}$ and is related to $u_{\star}$ by

$$
u_{\star}(x)=\frac{x-b_{j}}{\left|x-b_{j}\right|} e^{i H_{j}(x)}, \quad \text { near } b_{j} .
$$

Let

$$
R_{0}(x)=S_{j}(x)-\sum_{i \neq j} \log \left|x-b_{i}\right| .
$$

Our variant of the vanishing gradient property in [BBH4] (Corollary VIII.1) is:

THEOREM 3. - The following properties are equivalent:

i) $a=\left(a_{1}, \ldots, a_{d}\right)$ is a critical point of the renormalized energy $\widetilde{W}$.

ii) $\nabla S_{j}\left(a_{j}\right)=\frac{1}{4} \frac{\nabla w\left(a_{j}\right)}{w\left(a_{j}\right)}$, for each $j$.

iii) $\nabla H_{j}\left(a_{j}\right)=\frac{1}{4 w\left(a_{j}\right)}\left(-\frac{\partial w}{\partial x_{2}}\left(a_{j}\right), \frac{\partial w}{\partial x_{1}}\left(a_{j}\right)\right)$, for each $j$.

iv) $\nabla R_{0}\left(a_{j}\right)+\sum_{i \neq j} \frac{a_{j}-a_{i}}{\left|a_{j}-a_{i}\right|^{2}}=\frac{1}{4} \frac{\nabla w\left(a_{j}\right)}{w\left(a_{j}\right)}$, for each $j$.

The proof follows by the above considerations and the fact that, for each $j$,

$$
\nabla R_{0}(x)=\nabla S_{j}(x)-\sum_{i \neq j} \frac{x-a_{i}}{\left|x-a_{i}\right|^{2}}
$$




\section{SHRINKING HOLES AND THE RENORMALIZED ENERGY WITH WEIGHT}

As in [BBH4], Chapter I.4, we may define the renormalized energy by considering a suitable variational problem in a domain with "shrinking holes".

Let, as above, $G$ be a smooth, bounded and simply connected domain in $\mathbb{R}^{2}$ and let $b_{1}, \ldots, b_{k}$ be distinct points in $G$. Fix $d_{1}, \ldots, d_{k} \in \mathbb{Z}$ and a smooth data $g: \partial G \rightarrow S^{1}$ of degree $d=d_{1}+\ldots+d_{k}$. For each $\eta>0$ small enough, define

$$
G_{\eta}^{w}=G \backslash \bigcup_{j=1}^{k} \overline{\omega_{j, \eta}}
$$

where

$$
\omega_{j, \eta}=B\left(b_{j}, \frac{\eta}{\sqrt{w\left(b_{j}\right)}}\right)
$$

Set

$$
\mathcal{E}_{\eta}^{w}=\left\{v \in H^{1}\left(G_{\eta}^{w} ; S^{1}\right) ; \operatorname{deg}\left(v, \partial \omega_{j, \eta}\right)=d_{j} \text { and } v=g \text { on } \partial G\right\} .
$$

We consider the minimization problem

$$
\min _{u \in \mathcal{E}_{\eta}^{w}} \int_{G_{\eta}^{w}}|\nabla u|^{2}
$$

The following result shows that the renormalized energy $\widetilde{W}$ is what remains in the energy after the singular "core energy" $\pi d|\log \eta|$ has been removed.

THEOREM 4. - We have the following asymptotic estimate:

$$
\frac{1}{2} \int_{G_{\eta}^{w}}\left|\nabla u_{\eta}\right|^{2}=\pi\left(\sum_{j=1}^{k} d_{j}^{2}\right)|\log \eta|+\widetilde{W}(b, \bar{d}, g)+O(\eta), \quad \text { as } \eta \rightarrow 0,
$$

where

$$
\widetilde{W}(b, \bar{d}, g)=W(b, \bar{d}, g)+\frac{\pi}{2}\left(\sum_{j=1}^{k} d_{j}^{2} \log w\left(b_{j}\right)\right) .
$$


Proof. - As in [BBH4], Chapter I we associate to (26) the linear problem:

$$
\left\{\begin{array}{l}
\Delta \Phi_{\eta}=0, \quad \text { in } G_{\eta}^{w} \\
\Phi_{\eta}=C_{j}=\text { Const., } \quad \text { on each } \partial \omega_{j, \eta} \\
\int_{\partial \omega_{j, \eta}} \frac{\partial \Phi_{\eta}}{\partial \nu}=2 \pi d_{j}, \quad \text { for each } j=1, \ldots, k \\
\frac{\partial \Phi_{\eta}}{\partial \nu}=g \wedge g_{\tau}, \quad \text { on } \partial G \\
\int_{\partial G} \Phi_{\eta}=0 .
\end{array}\right.
$$

With the same techniques as in [BBH4] (see Lemma I.2), one may prove that

$$
\left\|\Phi_{\eta}-\Phi_{0}\right\|_{L^{\infty}\left(G_{\eta}^{w}\right)}=O(\eta)
$$

where $\Phi_{0}$ is the unique solution of (1).

Note that the link between $\Phi_{\eta}$ and an arbitrary solution $u_{\eta}$ of (26) is

$$
\left\{\begin{array}{l}
u_{\eta} \wedge \frac{\partial u_{\eta}}{\partial x_{1}}=-\frac{\partial \Phi_{\eta}}{\partial x_{2}} \quad \text { in } G_{\eta}^{w} \\
u_{\eta} \wedge \frac{\partial u_{\eta}}{\partial x_{2}}=\frac{\partial \Phi_{\eta}}{\partial x_{1}} \quad \text { in } G_{\eta}^{w}
\end{array}\right.
$$

From now on the proof follows the same lines as of Theorem I.7 in [BBH4].

\section{ACKNOWLEDGEMENTS}

We are grateful to Prof. H. Brezis for his encouragements during the preparation of this work.

\section{REFERENCES}

[BBH1] F. BETHUel, H. BREzis and F. Hélein, Limite singulière pour la minimisation des fonctionnelles du type Ginzburg-Landau, C. R. Acad. Sc. Paris, Vol. 314, 1992, pp. 891-895.

[BBH2] F. Bethuel, H. Brezis and F. Hélein, Tourbillons de Ginzburg-Landau et énergie renormalisée, C. R. Acad. Sc. Paris, Vol. 317, 1993, pp. 165-171. 
[BBH3] F. Bethuel, H. Brezis and F. Hélein, Asymptotics for the minimization of a Ginzburg-Landau functional, Calculus of Variations and PDE, Vol. 1, 1993, pp. 123-148.

[BBH4] F. Bethuel, H. Brezis and F. HÉLEIN, Ginzburg-Landau Vortices, Birkhäuser, 1994.

[LR] C. LEFTER and V. RăDulEsCu, On the Ginzburg-Landau energy with weight, $C . R$. Acad. Sc. Paris, Vol. 319, 1994, pp. 843-848.

[S] M. STRUwE, Une estimation asymptotique pour le modèle de Ginzburg-Landau, $C . R$. Acad. Sc. Paris, Vol. 317, 1993, pp. 677-680.

(Manuscript received June 10, 1994; revised version received July $11,1994$. 